\title{
NATURAL PERMEATION ENHANCER FOR TRANSDERMAL DRUG DELIVERY SYSTEM AND PERMEATION EVALUATION: A REVIEW
}

\author{
ASHA DAS*, ABDUL BAQUEE AHMED \\ Department of Pharmaceutics, Girijananda Chowdhury Institute of Pharmaceutical Science, Guwahati, Assam, India. \\ Email: ashagips@gmail.com
}

Received: 25 April 2017, Revised and Accepted: 20 May 2017

\begin{abstract}
The transdermal drug delivery route is evolving as a potential route due to its advantages of bypassing the hepatic first pass metabolism, decreased side effects and gastrointestinal effects, improve patience compliance as it is a pain-free self-administration for patients, etc. The major setback appearing in this route is the difficulty of the drugs to penetrate through the skin as the stratum corneum (outermost layer of the skin) forms a protective barrier for the underlying tissues from the outer environment. A transdermally delivered drug can only show its action when it can cross the transdermal barrier to reach the systemic circulation and for helping on doing that the penetration enhancer are the agents which increase the permeability of the skin which on return maintains the drug level in the blood. Permeation enhancers can be of a chemical type, natural type, and physical type. The present review describes the natural permeation enhancers can be which be employed for transdermal permeation of drugs.
\end{abstract}

Keywords: Transdermal drug delivery system, Permeation enhancer, Transdermal barrier.

(C) 2017 The Authors. Published by Innovare Academic Sciences Pvt Ltd. This is an open access article under the CC BY license (http://creativecommons. org/licenses/by/4. 0/) DOI: http://dx.doi.org/10.22159/ajpcr.2017.v10i9.19389

\section{INTRODUCTION}

Transdermal drug delivery offers a very advantageous route for drug delivery compared with the other routes of drug administration having advantages such as bypassing the hepatic first pass metabolism, and longer duration of action [1,2]. However, the barrier function of the skin outermost layer, the stratum corneum (SC) is one of the main limitations to it, and for this reason, skin penetration enhancers are gaining the greatest interest in pharmaceutical research [3].

Penetration enhancers help in the permeation of the desired drug (penetrant) through the skin by lowering the impermeability of the skin. Some properties which are desired in permeation enhancers are the must be pharmacologically inert, nonirritating, nontoxic, nonallergic, compatible with drugs and excipients, odorless, tasteless, colorless, and inexpensive and also have good solvent properties [4]

For a transdermal matrix patch to be successful, it relies on the capability of the penetrant (drug) to penetrate into the skin in quantities which is sufficient to maintain therapeutic levels. During formulating transdermal drug delivery system (TDDS), chemical enhancers are used widely as it helps in reducing the permeation barrier properties of the skin. A number of literature review mentioned that naturally occurring oils (essential oil and vegetable oil) can be employed as permeation enhancers due to their advantageous properties such as compatibility with drug and excipients, nontoxic, less allergic, and easy availability [5].

This review article describes the barrier properties of the skin and various natural penetration enhancers that have been employed to enhance the transdermal permeation of drugs and the different parameters for permeation evaluation.

\section{SKIN AS A BARRIER TO DRUG PERMEATION}

The outermost few microns of the skin, the SC, aids to the barrier function of the skin. This layer of the skin is the most impermeable, forming a laminate of compressed keratin-filled corneocytes attached in a lipophilic matrix. The lipids of this matrix are distinguishing in many respects:
- From the skin surface to the base of the SC, they provide the only continuous phase

- Among the biomembranes, the absence of phospholipid is particular and is a composition (ceramides, free fatty acids, and cholesterol) that is unique

- The SC lipids exist as multilamellar sheets even though it is having a deficit of polar bilayer forming lipids

- The essentially saturated, long-chain hydrocarbon tail aids a highly ordered, interlock configuration.

However, the resistivity of the membrane cannot be entirely explained by the unusual lipid matrix, and the architecture of the SC altogether has been proposed to play a role in the barrier property of the membrane. The corneocyte resembling a brick and mortar assembly is suggested to impart the membrane impermeable to water with respect to other biomembranes. And by the visualization studies localizing several permeants, in the intercellular channels by kinetic analysis of the in vivo skin penetration rates of model compounds and by evidence from thermotropic biophysical studies of lipid domains the transport role of this pathway gets furthermore support [6].

\section{Transdermal permeation pathways}

Transdermal permeation can take place by diffusion through:

A. Through the SC: By means of the transcellular route, drugs can pass through the corneocytes highly hydrated keratins are present in corneocyte, which provides an aqueous environment for which the hydrophilic drugs can pass. Hence, the transcellular pathway is a predominant pathway for hydrophilic drugs

B. Intercellular permeation: In intercellular pathway, the drug diffusing takes place by means of the continuous lipid matrix

C. Transappendaged permeation: Only $0.1 \%$ of the skin surface area is covered by the hair follicle and sweat glands which limit the area available for the applied drug formulation to come in contact with it. For many drugs, an aqueous pathway is considered desirable but as the sweat is traveling against the diffusion pathway of permeant, permeation may be limited. Lipid-rich sebum fills the sebaceous gland, which may present a barrier for hydrophilic drugs [7]. 


\section{PHYSICOCHEMICAL ASPECTS OF SKIN PENETRATION}

Diffusion of drug through the skin is a passive kinetic process which occurs down the concentration gradient (from a high concentration region to a low concentration region). The steady state equation can be described by Fick's first law of diffusion. The equation describes the rate of transfer (flux, J) of a diffusing substance through the unit area of the membrane and diffusion coefficient, $\mathrm{D}$ to the concentration gradient across the membrane $(\mathrm{dc} / \mathrm{dx})$.

$J=-A D(d c / d x)$

The negative sign in equation (1) is because the diffusion process occurs in the opposite direction to increased concentration. Fick's second law of diffusion, equation (2) can be derived from equation (1) to describe membrane transport under nonsteady state condition:

$d c / d t=D d 2 c / d x 2$

By maintaining the sink conditions in the receptor compartment and maximum fixed concentration in the donor compartment, the equation (2) can be written as:

$\mathrm{J}=\mathrm{AD}(\mathrm{Cm} / \mathrm{h})$

Where, $\mathrm{Cm}$ is the concentration in the donor-membrane interphase and $\mathrm{h}$ is effective diffusional path length. The $\mathrm{Cm}$ in the equation (3) can be used to replace by vehicle membrane partition coefficient (K) as the ratio between the concentration of permeant in the membrane at the donor-membrane interface and the vehicle in which applied (Cv). Modified Fick's first law of diffusion describes the steady-state flux across the membrane equation.

\section{$\mathrm{J} \mathrm{SS}=\mathrm{ADKCV} / \mathrm{h}$}

We can conclude that increased drug flux can be achieved by a change in $\mathrm{D}, \mathrm{K}$, and $\mathrm{C}$. The compounds which are skin penetration enhancers should potentially change the solubility or partition behavior of the drug into SC or its diffusion properties or both sometimes change in thermodynamic activity of drugs in the formulation manipulate the flux [8].

\section{PERMEATION ENHANCERS}

Permeation enhancers are the compounds which promote skin permeability [9]. They are an important factor in a TDDS which is used to improve the flux (J). Flux can be defined as the amount of material flowing through the unit cross section area at the time $(t)$ [10].

Ideal properties of penetration enhancers:

- They must be pharmacologically inert, nonallergic, nonirritating, and nontoxic

- It must have compatibility with excipients and drugs

- It must not have any pharmacological activity in the body

- Cosmetically it must be acceptable

- It must be odorless, tasteless, and colorless

- It should allow therapeutic agents into the body but should prevent the loss of endogenous material from the body, i.e., they should work unidirectionally

- It must have chemical and physical stability

- It must have a reproducible and predictable duration of action

- It must have a good solvent property $[11,12]$.

\section{NATURAL PERMEATION ENHANCERS (NPES)}

NPEs are comparatively new class of penetration enhancer in the pharmaceutical industry. Due to its advantages such as low cost, better safety profile more research need to be focused in this field to develop a stable transdermal formulations containing natural permeation enhancers (NPEs) which can be scale up for commercial transdermal drug product [13].

\section{Papain}

Papain is isolated from Carica papaya. It is an endocytic plant cysteine protease enzyme [14]. Papain, which is a proteolytic enzyme, was studied in vitro and in vivo permeation of low-molecular-weight heparin (LMWH). The combined administration of LMWH and papain was found to be a new approach in improvement in absorption of orally administered heparin and hence its bioavailability [15].

\section{Piperine}

Piperine is obtained from mature fruits of Piper nigrum and Piper longum [16]. Piperine was investigated for in vitro permeation of aceclofenac across human cadaver skin, and Fourier transform infrared technology was used to check the possible mechanism from which the results obtained showed that piperine enhances transdermal permeation of aceclofenac by biphasic mechanism involving partial extraction of SC lipid and interaction with SC keratin [17].

\section{Capsaicin}

Capsaicin is a major alkaloid among capsaicinoids which is produced only in capsicum fruits of the genus capsicum and belonging to the Solanaceae family [18].

The permeation enhancing properties of capsaicin were studied for naproxen taking azone as the standard enhancer and capsaicin were compared to it. Onto the skin, different amount of chosen enhancer was applied before experiment. A formulation containing $3 \%$ capsaicin and commercially available naproxen gel formulation was also studied, and the results were compared. It was found that penetration increased when the skin was treated with azone and capsaicin and also some alteration by capsaicin was seen in the SC layer. Hence, it was observed that capsaicin increases the penetration of naproxen through SC, which concludes that capsaicin is quiet capable enhancer of skin like wellknown enhancer azone [19].

\section{Myristica fragrans}

$M$. fragrans was evaluated as a penetration enhancer in a transdermal gel formulation containing diclofenac sodium as the target drug. Methanolic extracts, chloroform extracts, and n-hexane extracts of M. fragrans were used as a penetration enhancer in comparison to a synthetic enhancer Triton X. It was found that in both in vivo and in vitro studies, methanol and chloroform extract showed better percentage cumulative release (\%), and hence, better penetration was shown as compared to the synthetic enhancer [20].

\section{ESSENTIAL OIL}

Essential oil is natural products which are extracted from aromatic plants having a concoction of a number of aromatic smelling volatile compounds, primarily consisting compounds such as terpenes, terpenoids, and phenylpropanoids [21]. They can be accepted as a natural alternative to synthetic skin penetration enhancer due to their promising penetration enhancing activity [22].

As penetration enhancer, essential oils help in the delivery of drug compounds into the skin by interacting with the intercellular lipids by different physical processes such as increased disorder, phase separation, and fluidization. As they are penetrated easily by the skin, they are easily excreted also by the body with urine and feces. Hence, due to their better safety profile in comparison with other penetration enhancers, their use is increasing [23].

\section{Essential oil as skin permeation enhancer}

Penetration enhancer interacts with the tissue components to lessen the barrier properties by partitioning into the SC but do not cause any damage to the underlying skin cell. Both D-limonene and 1, 8-cineole have shown to modify permeant diffusivity by disrupting SC lipid [24].

\section{Eucalyptus oil}

The oil of eucalyptus can be obtained from a number of species of the Myrtaceae family, such as Eucalyptus citriodora, Eucalyptus dives, 
Eucalyptus globulus, Eucalyptus polybractea, and Eucalyptus radiata. By steam distillation of eucalyptus leaves, its oil is extracted. The eucalyptus oil was subjected to permeation studies on full thickness human skin and it was found that the oil enhanced the permeation of chlorhexidine $(2 \%[\mathrm{w} / \mathrm{v}])$ into the dermis and the lower layer of the epidermis when it was combined with $70 \%(\mathrm{w} / \mathrm{v})$ isopropyl alcohol and $10 \%(\mathrm{v} / \mathrm{v})$ eucalyptus oil in comparison to the solution of chlorhexidine/isopropyl alcohol alone [25].

\section{Niaouli oil}

The extraction of niaouli oil can be made by steam distillation of twigs and leaves of Melaleuca quinquenervia, of the family Myrtaceae. The major constituents of niaouli oil was 55-70\% 1,8-cineole (oxide) and limonene (monoterpene), 7-15\% a-pinene (monoterpene), 2-6\% s-pinene (monoterpene), and $2-6 \%$ viridiflorol (sesquiterpene). In vitro studies were performed to determine the permeation enhancing the effect of niaouli oil at $10 \%(\mathrm{w} / \mathrm{w})$ concentration in propylene glycol on estradiol model drug using a hairless mouse skin. It was found that niaouli oil proved to be more effective in transdermal permeation of estradiol than cajput, myrtle, orange, and cardamom essential oil [26].

\section{Fennel oil}

The extraction of fennel oil can be made from the seeds of Foeniculum vulgare, of the family Umbelliferae. On permeation studies, the percutaneous penetration of trazodone hydrochloride was enhanced by fennel oil followed by eucalyptus oil, citronella oil, and mentha oil. The variable physicochemical properties and molecular weights of phytochemicals present in the different essential oils may be the factors for the differences in the permeation enhancement activity between the oils [27].

\section{Black cumin oil}

The extraction of black cumin oil is made by steam distillation of the seeds of Cuminum cyminum by steam distillation. Black cumin oil showed a relatively greater permeating effect for the drug carvedilol when it was compared to clove oil, eucalyptus oil, tulsi oil, oleic acid, Tween 80 and the enhancement factor was found to be 6.40 . Furthermore, Fourier transform infrared spectroscopy studies confirmed the alteration caused by black cumin oil on the permeability of the skin by extracting lipids and by hydrogen bonding which affects other hydrogen bonds between the ceramides [28].

\section{Almond oil}

Oil of almond and oleic acid were found as promising carriers/vehicles for enhanced permeability and solubility of aceclofenac. Hence, these oils can be used to develop drug delivery systems for improved bioavailability of aceclofenac [29].

In the study, topically applied ketoprofen gels and patches were formulated and evaluated, and almond oil was checked as a penetration enhancer for ketoprofen gels and patches through artificial membrane/ rabbit skin. It was found that almond oil in different concentration as a penetration enhancer enhances the penetration of drugs from transdermal gels and patches across synthetic membrane/rabbit skin but notably when used in $3 \%$ concentration [30].

\section{Basil oil}

Basil oil was studied for its potential as a permeation enhancer for labetalol hydrochloride with respect to camphor, thymol, geraniol, and clove oil. It was suggested that basil oil is having good penetration enhancing property for improved transdermal drug delivery of labetalol [31].

Basil oil was used for the enhancement in bioavailability of flurbiprofen applied transdermally and it was concluded that the bioavailability of the flurbiprofen applied transdermally using basil oil increased by 2.97 , 3.80 , and 5.56 times in comparison to the flurbiprofen administered orally in albino rats [32].

\section{Alpinia oxyphylla oil}

A. oxyphylla oil was extracted from A. oxyphylla and was divided into a higher polarity fraction and a lower polarity fraction. In vitro studies were performed using Franz diffusion cell across the dorsal skin of Wistar rats, the results indicated that the high polarity fraction of A. oxyphylla oil was having more efficient permeation enhancing the effect of indomethacin at concentration $3 \%$ and $5 \%$ then the lower polarity fraction [33].

\section{Turpentine oil}

Turpentine oil on the skin permeation rate of flurbiprofen showed an additive effect when it was added to an optimized cosolvent mixture of propylene glycol and isopropyl alcohol $(30-705[\mathrm{v} / \mathrm{v}])$, and at the concentration of $5 \%(\mathrm{v} / \mathrm{v})$ of turpentine oil, maximum transdermal penetration rate was obtained [34].

The effectiveness of turpentine oil was investigated for permeation enhancing activity for diclofenac dimethylamine matrix patches across the artificial skin in the Franz diffusion cell. It was found that the oil showed increasing permeation with increasing concentration of turpentine [35].

\section{Rosemary oil}

Rosemary oil is extracted from Rosmarinus officinalis. Rosemary oil when investigated for skin permeation enhancing activity for diclofenac sodium topical gel showed enhanced skin absorption at $0.5 \%$ and $1 \%$ concentration, respectively [36].

\section{Cardamom oil}

Cardamom (Elettaria cardamomum) is a common spice of India belonging to the Zingiberaceae family [37]. The oil extracted from cardamom has a number of volatile compounds such as monoterpenes including 1,8-cineole and cis-ocimene and sesquiterpene including guanine and nerodilol [38]. Cardamom oil on in vitro permeation studies through the rabbit abdominal skin showed an increase in penetration of the drugs indomethacin, diclofenac, and piroxicam [39].

\section{TERPENES}

In transdermal drug delivery studies, terpenes are one of the major choices. This class includes a heterogeneous range of members. The physicochemical properties of a specific terpene play a role in showing the effect on the skin, in particular, its lipophilicity. However, smaller terpenes with nonpolar groups are said to be better skin permeation enhancers [40]. Terpenes are also reported to increase drug partitioning and diffusibility into the skin by disturbing the lipid bilayers of the skin [41]. They are relatively safe as skin penetration enhancers for both hydrophilic and lipophilic drugs [42].

\section{Farnesol}

Farnesol is present in many essential oils, such as citronella, neroli, cyclamen, lemongrass, tuberose, balsam, and tolu. It is a sesquiterpene alcohol. Farnesol $(0.25 \%)$ was reported to increase the permeation of diclofenac sodium with respect to other terpenes in the following order: Farnesol $>$ carvone $>$ nerolidol $>$ menthone $>$ limonenoxide [43].

\section{Menthol}

Menthol, which is one of the potent penetration enhancers, is obtained from the flowering tops of Mentha piperita. Menthol and limonene together can be used as a prototype of terpenes that can be used as permeation enhancer [44].

\section{Eucalyptol}

Eucalyptol is a cyclic ether and a monoterpenoid known by a number of synonyms such as 1, 8-cineole, cajeputol, eucalyptol, and cineole. Eucalyptol is used in cosmetic, fragrances and flavoring industries because of its spicy aroma and taste. 1, 8-cineole has also been used for the percutaneous absorption of several lipophilic drugs through the hairless mouse skin [45].

\section{Eugenol}

Eugenol was evaluated for permeation enhancing effect for the drug lornoxicam which is a nonsteroidal anti-inflammatory drug of oxicam class. Lornoxicam transdermal patches were formulated which were 
then subjected to in vitro studies in a Franz diffusion cell using rat skin. In vitro studies showed that eugenol does increase the permeation of lornoxicam across rat skin [46].

\section{Borneol}

The transdermal permeation enhancing activity of borneol was investigated on 5 model drug, namely, 5-fluorouracil, antipyrine, aspirin, salicylic acid, and ibuprofen and it was found that borneol effectively promoted the transdermal permeation of the model drugs [47].

\section{PERMEATION STUDIES}

The estimation of percutaneous absorption of a molecule is a very important step in the evaluation of a molecule of TDDS [48]. Moreover, in both pharmaceutical and cosmetic research, to support the development of TDDS skin permeation rates, evaluation has become a very important step [49]. The various ways by which the transdermal permeations of a drug molecule can be studied are.

\section{In vitro release studies}

Franz diffusion cell is used for carrying out in vitro release studies wherein the donor compartment the circular patch is mounted, the receptor compartment of which is filled with phosphate buffer saline $(\mathrm{pH}-7.4)$, and the whole assembly is maintained at $32^{\circ} \mathrm{C}$ by the warm water circulation through the water jacket and is stirred at $40-50 \mathrm{rpm}$. The patch was kept in contact with the receptor liquid. About $0.5 \mathrm{ml}$ of sample was withdrawn at regular intervals for $8 \mathrm{hrs}$ and was replaced with the same amount of the medium. The samples were subjected to filtration, diluted, and analyzed spectrophotometrically [50].

\section{In vivo release studies}

\section{Animal models}

Hairless rat, hairless rhesus monkey, guinea pig, and rabbit are the most commonly used species used for evaluating transdermal drug. One of the most reliable models for in vivo evaluation of transdermal delivery in man is the rhesus monkey [51]. Animals in small scale are preferred for in vivo studies because of economic factors and availability [52].

\section{Human volunteers}

Clinical phase is one of the important stages in the development of a transdermal device, and in this stage, the formulation is tested in human volunteers for the collection of pharmacokinetic and pharmacodynamics data which are required to evaluate the generation of toxic effect during application of formulation. C14 radioisotope is used for labeling of drug to determine percutaneous absorption in human and measuring the radioactivity in excreta, but for knowing, amount residing in a body and amount excreted by other route attention is required [52].

\section{In vivo dermal absorption study}

For in vivo dermal absorption test, the dorsal surface (approximately $4 \mathrm{~cm} \times 3 \mathrm{~cm}$ area) of the rabbit skin is first clipped free for the application of the test substance and this is done before 24 hrs of starting the test. The requisite amount of the test substance was applied to the area, and $1 \mathrm{ml}$ of blood sample was withdrawn from the central ear artery at predetermined times over $24 \mathrm{hrs}$ at 2, 6, 12, $24 \mathrm{hrs}$. Plasma was obtained by centrifuging the blood and was stored at $-20^{\circ} \mathrm{C}$ before analysis. The analysis was done using headspace gas chromatographymass spectrometry [53].

\section{CONCLUSION}

The field of transdermal drug delivery due to its advantages has been rapidly developing and which has stimulated various researches to incorporate more and more drugs through transdermal route. Skin serves a limitation for permeation of drugs, and hence, permeation enhancers are used to increase the permeability of the poorly absorbed drugs and hence maintain its bioavailability. The review article summarizes the various NPEs which can be used to accelerate the permeation of drug across skin for the development of transdermal delivery system and the parameters by which the permeation studies can be done. In this article, various NPEs has been discussed which can be used to accelerate the permeation of drug across skin for development of TDDS.

\section{REFERENCES}

1. Subhash PG, Dinesh BM, Ravikumar M. Feasibility of lercanidipine $\mathrm{Hcl}$ for TDDS: Permeation kinetics study in presence of various penetration enhancers. Int J Pharm Pharm Sci 2011;3:103-7.

2. Ravichandiran V, Manivannan S. Wound healing potential of transdermal patches containing bioactive fraction from the bark of Ficus racemose. Int J Pharm Pharm Sci 2015;7:326-32.

3. Chantasart D, Li SK. Structure enhancement relationship of chemical penetration enhancers in drug transport across the stratum corneum. Pharmaceutics 2012;4:71-92.

4. Jagannath SS, Manohar SD, Bhanudas RB. Chemical penetration enhances - A review. World J Pharm Pharm Sci 2014;3(2):1068-80.

5. Patel JK, Jani RK. Enhancing effect of natural oil as permeation enhances for transdermal delivery of diltiazem hydrochloride through wistar rat skin. Int J Pharm Sci Rev Res 2016;36(1):9-16.

6. Naik A, Kalia YN, Grey RH. Transdermal drug delivery: Overcoming the skin's barrier function. Pharm Sci Technol Today 2000;3(9):318-26.

7. Ramani R, Pandya S, Motka U, Lakhani D, Ramaney D, Sheth D. Drug penetration enhancement in transdermal drug delivery system by chemical penetration enhancers. Int J Preclin Pharm Res 2013;4(1):10-7.

8. Raut SV, Nemade LS, Desai MT, Bonde SD, Dongare SU. Chemical penetration enhancers: For transdermal drug delivery systems. Int J Pharm Res Rev 2014;4(1):33-40.

9. Raza R, Ashu M, Kumar P, Alam S, Prakash S, Chauhan N. Approaches and evaluation of transdermal drug delivery. Int $\mathrm{J}$ Drug Dev Res 2015;7(1):222-33

10. Dhamecha D, Rathi AA, Saifee M, Lahoti SR, Dehghan HG. Drug vehicle based approaches of penetration enhancement. Int J Pharm Pharm Sci 2009;1(1):24-46.

11. Jaydatt KJ, Sreenivas SA. Review on chemical permeation enhances used in transdermal drug delivery system. Int J Sci Innov Discov 2012;2:204-17.

12. Pfister WR, Hsieh DS. Permeation enhancers compatible with transdermal drug delivery systems. Part I: Selection and formulation considerations. Med Device Technol 1990;1:48-55.

13. Lahor DW, Chaudhary V, Shah KS. Terpenes: Natural skin penetration enhances in transdermal drug delivery system. Int J Pharm Res Dev 2011;2(12):39-45.

14. Amri E, Mamboya F. Papain, a plant enzyme of biological importance: A review. Am J Biochem Biotechnol 2012;8(2):99-104.

15. Gabovac V, Schmit ZT, Bernkop SA. Papain: An effective permeation enhances for orally administered low molecular weight heparin. J Pharm Res 2007;24(5):1001-6.

16. Prakash S, Kherde P, Rangari V. Bioenhancement effect of piperine and ginger oleo resin on the bioavailability of atazanvir. Int J Pharm Pharm Sci 2015;7(10):241-5.

17. Shah KK, Shiradkar MR, Bindu VH. Transdermal drug delivery of acleofenac: Effect of piperine and its mechanism of action. Int J Pharm Bio Sci 2011;2(3):3-7.

18. Arora R, Gill NS, Chauhan G, Rana AC. An overview about versatile molecule capsaicin. Int J Pharm Sci Drug Res 2011;3(4):280-6.

19. Saini S, Chauhan SB, Agarwal SS. Recent development in transdermal drug delivery system. J Adv Pharm Educ Res 2014;4(1):31-40.

20. Patwardhan SK, Bhinde MA. Evaluation of Myristica fragnansas a penetration enhancer in transdermal gel formulation. Int J Pharm Pharm Sci $2015 ; 7(3): 350-5$.

21. Cheng J, Jiang QD, Wee YH, Liu P, Yao JH, Lu Q, et al. Potential of essential oils as penetration enhances for transdermal administration of ibuprofen to treat dysmenorrhea. Molecules 2015;20:18219-36.

22. Majumder M, Sharma HK, Zaman K, Lyngdoh W. Evaluation of physicochemical properties and antibacterial activity of the essential oil obtained from the fruits of Zanthoxyllum acanthopodium DC collected from Meghalaya, India. Int J Pharm Pharm Sci 2014;6(5):543-6.

23. Herman A, Herman AP. Essential oils and their constituents as skin penetration enhances for transdermal drug delivery: A review. J Pharm Pharmacol 2014;67:473-85.

24. Aggarwal S, Agarwal S, Jalhan S. Essential oil as novel human skin penetration enhances for transdermal drug delivery: A review. Int J Pharm Bio Sci 2013;4(1):857-68

25. Karapen TJ, Conway BR, Worthington T, Hilton AC, Elliott TS, 
Lambert PA. Enhanced chlorhexidine skin penetration with eucalyptus oil. Bio Med Cent BMC Infect Dis 2017;10:278.

26. Monti D, Chetoni P, Burgalassi S, Najarro M, Fabrizio SM, Boldrini E. Effect of different terpene-containing essential oils on permeation of estradiol through hairless mouse skin. Int J Pharm 2002;237:209-14.

27. Das MK, Bhattacharya A, Ghoshal SK. Effect of different terpene containing essential oil on percutaneous absorption of trazodone hydrochloride through mouse epidermis. J Drug Deliv 2006;13:425-31.

28. Amin S, Kohli K, Khar RK, Mir SR, Pillar K. Mechanism of in vitro percutaneous absorption enhancement of carvedilol by penetration enhances. Pharm Dev Technol 2008;13(6):533-9.

29. Malik MZ, Ahmad M, Minhar MU, Munir A. Solubility and permeability studies of aceclofenac in different oils. Trop J Pharm Res 2014;13(3):327-30.

30. Goswami D, Uppal N, Goyal S, Mehta N, Gupta AK. Permeation enhances for TDDS from natural and synthetic sources: A review. J Biomed Pharm Res 2013;2(1):19-29.

31. Jain R, Aquil M, Ali A, Khar RK. Basil oil is a promising skin penetration enhances for transdermal drug delivery of labetotol hydrochloride. Drug Dev Ind Pharm 2008;34(4):384-9.

32. Kumar A, Aggarwal G, Singh K, Harikumar SL. Comparison of vegetable and volatile oils as skin permeation enhances for transdermal delivery of losartan potassium. Pharm Lett 2014;6(1):199-213.

33. Fang JY, Leu YL, Hueang TL, Chen HC, Hung CF. Development of sesquiterpenes from Alpinia oxyphylla as novel skin permeation enhances. Eur J Pharm Sci 2003;19(4):53-262.

34. Fort LT, Gerber M, Plessis JD, Hamman JH. Transdermal drug delivery enhancement by compounds of natural origin. Molecules 2011;16:10507-40

35. Khan NR, Khan GM, Khan AR, Wahab A, Asghar MJ, Akhlaq M, et al. Formulation, physical, in vitro and ex vivo evaluation of diclofenac diethylamine matrix patches containing turpentine oil as penetration enhancer. Afr J Pharm Pharmacol 2012;6(6):434-9.

36. Akbari J, Saeedi M, Farzin D, Semnani KM, Esmaili Z. Transdermal absorption enhancing effect of the essential oil of Rosmarinus officinalis on percutaneous absorption of $\mathrm{Na}$ diclofenac from topical gel. Pharm Biol 2015;53(10):1442-7.

37. Shetty P, Rao SN, Rani M. Preliminary phytochemical screening of ethanolic extract of Elettaria cardamomum fruits. Int J Univ Pharm Bio Sci 2013;2(6):584-90.

38. Husain SS, Ali M. Analysis of volatile oil of the fruits of Eletteria cardamomum (L) Maton and its antimicrobial activity. World J Pharm Pharm Sci 2014;3(2):1798-808.
39. Huang YB, Wu PC, Ko HM, Tsai YH. Cardamom oil as a skin permeation enhancer for indomethacin, piroxicam, and diclofenac. Int $\mathrm{J}$ Pharm 1995;126(1-2):111-7.

40. Karande P, Mitragotri S. Enhancement of transdermal drug delivery via synergistic action of chemicals. Biochem Biophys Acta 2009; 1788(11):2362-73

41. Jantarat C. Bioavailability enhancement techniques of herbal medicine: A case example of curcumin. Int J Pharm Pharm Sci 2013;5(1):493-500.

42. Gao S, Singh J. In vitro percutaneous absorption enhancement of a lipophilic drug tamoxifen by terpenes. J Control Release 1998;51(2-3):193-9.

43. Nokhodchi A, Sharabiani K, Pashidi MR, Ghafourian T. The effect of terpene concentrations on the skin penetration of diclofenac sodium. Int J Pharm 2007;335(1-2):97-105.

44. Singla V, Saini S, Singh G, Rana AC, Joshi B. Penetration enhancers: A novel strategy for enhancing transdermal drug delivery. Int Res J Pharm 2011;2(12):32-6.

45. Patel P, Patel P. Herbal excipients: An emerging field as a penetration enhances in transdermal drug delivery system. Int J Pharm Res Dev 2011;4(2):58-68.

46. Soujanya C, Satya LC, Reddy ML, Manogna K, Prakash PR, Ramesh A. Formulation and in vitro \& in vivo evaluation of transdermal patches of lornoxicam using natural permeation enhancers. Int J Pharm Pharm Sci 2014;6(4):282-6

47. Yi QF, Yan J, Tang SY, Huang H, Kang LY. Effect of borneol on the transdermal permeation of drugs with differing lipophilicity and molecular organization of stratum corneum lipids. Drug Dev Ind Pharm 2016;42(7):1086-93.

48. Godin B, Touitou E. Transdermal skin delivery: Prediction for humans from in vivo, ex vivo and animal models. Adv Drug Deliv Rev 2007;59(11):1152-61.

49. Gabor V, Szilvia B, Toth G, Balogh R, Csanyi E, Sinko B, et al. Permeability test for local therapeutic patches using skin PAMPA method. Eur J Pharm Sci 2015;76:165-72.

50. Rajabalaya R, Cheng DS, David SR. Development of transdermal ondansetron hydrochloride for the treatment of chemotherapy - Induced nausea and vomiting. Trop J Pharm Res 2013;12(3):279-85.

51. Patel D, Chaudhary SA, Parmar B, Bhura N. Transdermal drug delivery system: A review. Pharm Innov 2012;1(4):66-75.

52. Sachan R, Bajpai M. Transdermal drug delivery system: A review. Int J Res Dev Pharm Life Sci 2013;3(1):748-65.

53. Pednekar PP, Dhumal RV, Datar AG, Vanage GR. In vivo dermal absorption and sub-acute toxicity studies of essential oil from Blumea eriantha DC. Int J Pharm Pharm Sci 2013;5(3):351-8. 